\title{
Modelling of Oxygen Diffusion and Segregation at Interfaces in Ag-MgO Composites
}

\author{
I.V. Belova ${ }^{1, a}$ and A. Öchsner ${ }^{2, c}$, N. Muthubandara ${ }^{1}$ and G.E. Murch ${ }^{1, b}$
}

${ }^{1}$ Diffusion in Solids Group, Centre for Geotechnical and Materials Modelling, School of Engineering, The University of Newcastle, Callaghan, NSW 2308, Australia

${ }^{2}$ Department of Materials Engineering, Universiti Teknologi Malaysia, 81310 UTM Skudai, Johor, Malaysia

\author{
aIrina.Belova@newcastle.edu.au, ${ }^{b}$ Graeme.Murch@newcastle.edu.au, \\ coechsner@fkm.utm.my
}

Keywords: Segregation, diffusion, finite element method, Monte Carlo, interfaces, composites

\begin{abstract}
The presence of atomic oxygen at internal metal-ceramic oxide interfaces significantly affects the physical properties of the interfaces which in turn affects the bulk properties of the material. This problem is addressed for the model composite system $A g-M g O$ from a phenomenological point of view using a lattice-based Monte Carlo method and a finite element method extended with special user-subroutines. We simulate the time dependence of oxygen depth and contour profiles. We are able to show very good agreement between these two methods.
\end{abstract}

\section{Introduction}

The nature and integrity of the internal interfaces (interphase boundaries) between a metal matrix phase and a dispersed ceramic phase is of great importance in many technologies including those involving thin solid films, coatings, electronic packaging, supported catalysts, fibre-reinforced composites and dispersion-hardened materials (cermets). Some of these structures are formed by allowing oxygen to diffuse interstitially into a metallic alloy, one component of which preferentially oxidizes to form the dispersed ceramic phase. Further oxygen coming at the time of formation of the composite or later during high temperature service of the material can segregate to the internal metal-ceramic interfaces, changing their physical properties and ultimately adversely affecting the bulk properties of the composite. It is thus important to establish means for predicting the timedependence of the concentration of oxygen that segregates at the various interfaces of the material. Recently, the time-dependence of the diffusion of oxygen into the composite and the subsequent segregation of oxygen at $\mathrm{Ag}-\mathrm{MgO}$ interfaces (a model system) has been studied theoretically using simple one-dimensional finite difference models [1]. The principal input parameters to these calculations are the diffusivity of oxygen in $\mathrm{Ag}$ and an estimate of the segregation factor [2] of oxygen at the $A g-M g O$ interfaces. The influence of effects such as kinetic order or nonhomogeneity of the ceramic oxide inclusions was investigated. Later, more sophisticated geometries and particle distributions were investigated with a two-dimensional finite element scheme making use of commercial code that had been extended with special user-subroutines [3]. This numerical approach allowed for the consideration of somewhat more general boundary conditions, specimen sizes and time or concentration dependent material and materials process parameters. 
However, because of meshing difficulties, finite element schemes are restricted to quite simple particle distributions and boundary conditions. An alternative approach to the problem is the use of Monte Carlo methods. In the past, Monte Carlo methods have generally been viewed as being much too computationally demanding to be used for addressing phenomenological heat and mass transport problems. Recent advances in affordable computational resources now make the Monte Carlo method a viable alternative for the much more established methods such as the finite element method. Recently, a lattice-based Monte Carlo method that makes use of virtual random walking particles has been proposed to address phenomenological mass and thermal diffusion problems $[4,5]$. This method, which can be considered a convenient simulation form of a finite-difference method, conveniently copes with multi-scale modelling in both space and time. The method has enabled effective mass and thermal diffusivities/conductivities in two and three-phase composite materials to be readily calculated for various inclusion geometries and matrix and inclusion diffusivities [4,5]. It has also permitted the calculation of 'depth' concentration profiles of the diffusant such as tracer concentration depth profiles from the well-known thin-film or instantaneous tracer source condition for such problems as diffusion in the presence of grain boundaries, segregation of the diffusant to the grain boundary and within nanocrystalline material. Commercial software is not available at the present time for utilizing the Monte Carlo method for addressing phenomenological heat and mass transport problems. Indeed, the method is still in the relatively early stages of development for such applications. In the present paper, we address the phenomenological problem of the time-dependent segregation of oxygen at metal-ceramic $(A g-$ $\mathrm{MgO}$ ) interfaces by making use of a lattice-based Monte Carlo method. We address a simple geometric distribution of inclusions so that we are able to make a side-by-side comparison of the results with results using the finite element method.

\section{Theory}

The model. We consider the problem in $2 \mathrm{D}$ as shown in Fig. 1. The outer surface boundary is denoted by $\Gamma_{0}$. The surface concentration of oxygen at $\Gamma_{0}$ is kept constant for all times. The oxygen is permitted to diffuse into the $\mathrm{Ag}$ matrix denoted by region $\Omega_{1}$. Each square inclusion of $\mathrm{MgO}$ (indicated by $\Omega_{3}$ ) is surrounded by a very thin interface region $\Omega_{2}$ where the oxygen can adsorb/desorb i.e. to segregate. The inclusion, denoted by region $\Omega_{3}$, is considered itself to be impermeable to oxygen at the temperatures of interest.

The interface boundaries are $\Gamma_{1}$ - this is the boundary between regions $\Omega_{1}$ and $\Omega_{2}$ and $\Gamma_{2}-$ this is the boundary between regions $\Omega_{2}$ and $\Omega_{3}$. The square inclusions themselves are arranged in a square planar pattern in the present calculations but other shapes and arrangements can be readily employed. The model is assumed to be periodic in the direction normal to the diffusion direction (periodic boundary conditions are set at $\Gamma_{0}^{1}$ ). We also assume that the boundary $\Gamma_{0}^{2}$ is far enough from the $\Gamma_{0}$ so the concentration of oxygen at this boundary is always zero.

I. In region $\Omega_{1}$ we have for the oxygen concentration $\psi_{O}^{1}$ that:

$$
\frac{\partial \psi_{O}^{1}}{\partial t}=D_{O}^{1} \nabla^{2} \psi_{O}^{1}
$$

where $D_{O}^{1}$ is the diffusion coefficient of oxygen in the $A g$ matrix and $\nabla^{2}$ is the Laplacian operator. 


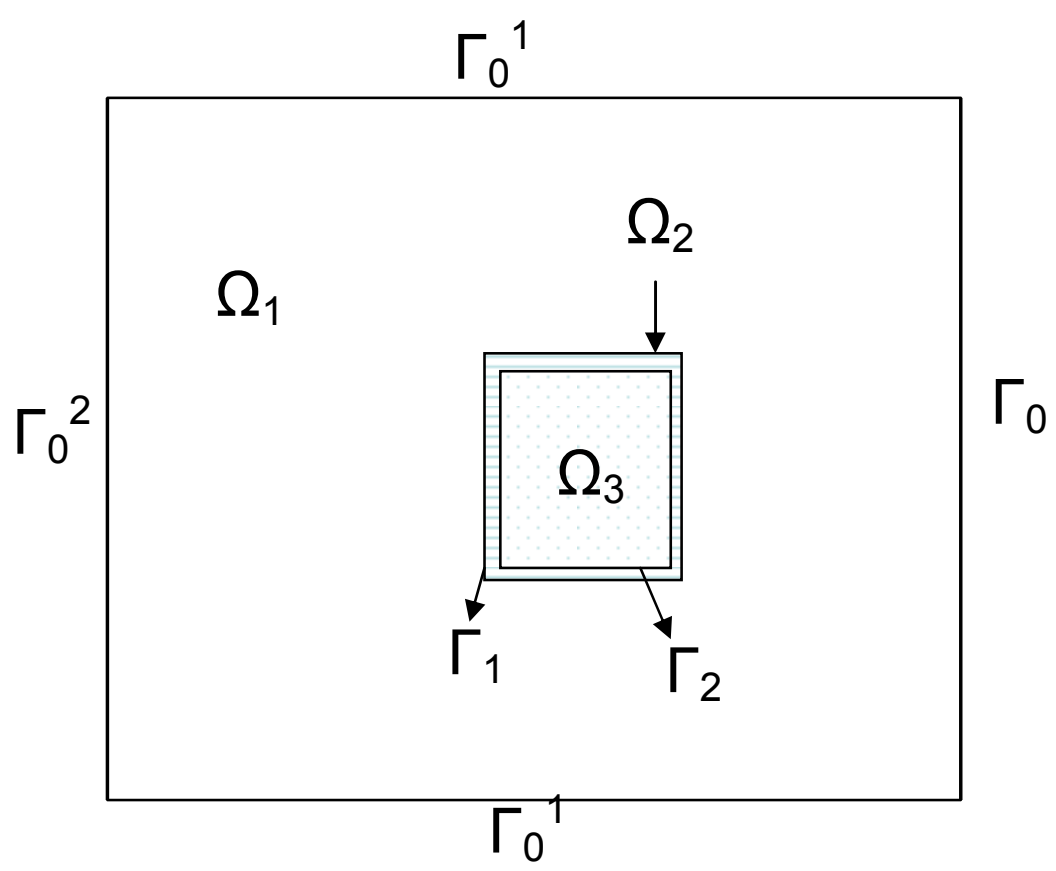

Figure 1. Schematic representation of the model for the adsorption/desorption (segregation) of oxygen at internal interfaces after diffusion through the matrix from an external surface $\Gamma_{0}$.

II. In region $\Omega_{2}$ we have for the oxygen concentration $\psi_{O}^{2}$ (we assume that this concentration is equivalent to the coverage, this is the $2 \mathrm{D}$ analogue of the $3 \mathrm{D}$ concentration) that:

$$
\frac{\partial \psi_{O}^{2}}{\partial t}=\operatorname{div}\left(D_{O}^{2} \nabla \psi_{O}^{2}\right)
$$

In principle, $D_{O}^{2}$ can take a different value along and perpendicular to $\Gamma_{1}\left(\Gamma_{2}\right)$. It can take any (constant) value when oxygen moves along the interface itself. We have put this intra interface mobility equal to zero; in other words, the oxygen can adsorb and desorb at the interface but not move along the interface.

III. In region $\Omega_{3}$ we have for the oxygen concentration $\psi_{O}^{3}$ that:

$$
\psi_{O}^{3}=0
$$

For the boundary condition at the outer boundaries we have the well-known constant concentration (or constant source) boundary condition:

$$
\psi_{O \mid \Gamma_{0}}^{1}=T_{1}=\text { const }
$$

at all times $t \geq t_{0}, t_{0}$ is the initial time.

For the inner boundaries we have the following conditions for the concentrations and fluxes: 
At $\Gamma_{1}$ we have that:

$$
\psi_{O \mid \Gamma_{1}}^{1} \neq \psi_{O \mid \Gamma_{1}}^{2},
$$

(At steady-state or at very long diffusion times for interfaces very close to the surface this will be $s \psi_{O \mid \Gamma_{1}}^{1}=\psi_{O \mid \Gamma_{1}}^{2}$ where $s$ is the segregation factor (Henry segregation isotherm [2]) and is known or can be estimated before the calculation. In principle, the oxygen can saturate the interface regions and, in effect, $s$ then depends on $\psi_{O}^{2}\left(\psi_{O}^{1}\right)$. Then the 'McLean segregation isotherm' [2] would be the appropriate one to employ. In the present paper, however, we have restricted ourselves to Henry-type isotherms.

For the flux then we have that:

$$
D_{O}^{1} \frac{\partial \psi_{O}^{1}}{\partial \vec{n}_{\Gamma_{1}}}=J_{O \mid \Gamma_{1}}^{2}, \quad \frac{\partial \psi_{O}^{2}}{\partial t}=\psi_{O}^{1} w_{12}-\psi_{O}^{2} w_{21}
$$

where $w_{12}, \quad w_{21}$ are local transition rates (see further below) which relate to the equilibrium segregation factor $\mathrm{s}$ :

$$
s=w_{12} / w_{21}
$$

At $\Gamma_{2}$ we have that:

$$
\psi_{O \mid \Gamma_{1}}^{2}=\psi_{O \mid \Gamma_{2}}^{2}
$$

and

$$
J_{O \mid \Gamma_{2}}^{2}=0 .
$$

The initial conditions are trivial: $\psi=0$ everywhere.

Method of computation. The method of computation using the finite element method has been described in detail elsewhere [1,2]. In the following section, we confine ourselves to describing the lattice-based Monte Carlo procedure along with some practical details. The regions $\Omega_{1}$ and $\Omega_{3}$ are mapped onto a fine grained square planar lattice of mesh size $a$. A large number of particles $\left(10^{5}\right)$ are released all at once at time $t=0$ from random positions chosen at the surface $\left(\Gamma_{0}\right)$ of the lattice. These particles are permitted to diffuse according to a hopping model by being chosen randomly to jump in a random direction and at a rate depending on their location as reflected in a transition rate $w$. The motion of the particles is restricted to a square planar lattice (in the present case $400 \times 50$, the former dimension is the diffusion direction) where the particles move on random walks for a given 'diffusion anneal' time $t$ (which is scaled to an attempt to jump). Typically, this time comprises some $10^{8}-10^{11}$ attempts to jump. Multiple occupancy of a site is permitted and accordingly there are absolutely no diffusion correlation effects in this problem. The diffusion coefficient $D_{\mathrm{O}}{ }^{1}$ of oxygen in the matrix $\Omega_{1}$ is represented by:

$$
D_{\mathrm{O}}{ }^{1}=w_{1} a^{2} / 4
$$

where $w_{1}$ is the inter-site transition rate in the matrix region $\Omega_{1}$ and $a$ is the lattice spacing (mesh size) and the factor 4 comes from the fact that the simulation is in $2 \mathrm{D}$ (this factor would be 6 for 3D). 
We denote a transition rate $w_{12}$ from the matrix region $\Omega_{1}$ to the interface region $\Omega_{2}$ (see further below). (This can be regarded as an adsorption process of oxygen to the interface. Similarly, we have the reverse of this, a transition rate $w_{21}$ from the interface region $\Omega_{2}$ back to the matrix region $\Omega_{1}$. (This can be regarded as a desorption process of oxygen from the interface to the matrix.) As mentioned above, in our calculations the oxygen mobility along the interface in this problem was suppressed as well as the oxygen mobility within the inclusion itself. The highest transition rate (say $w_{1}$ ) is scaled to unity for computational efficiency.

A constant source of particles at the surface (plane 201) for all diffusion times is obtained in the following way. The idea is simply to keep the number of virtual particles constant on this surface plane at, say, $10^{5}$ for all diffusion times. As each particle leaves the source plane, it is immediately replaced by a new one, which is again generated at a random position on this plane in order to maintain the number designated $\left(10^{5}\right)$. On the other hand, whenever a particle returns to the source plane, thereby exceeding the number designated $\left(10^{5}\right)$ then that particle is permanently removed from the system. Over a period of time, as the material absorbs more diffusant, the number of particles in the system naturally increases. Accordingly, the diffusion time $t$ needs to be constantly re-scaled since it is proportional to the number of attempts per particle.

It is useful to discuss the meaning of 'distance' in the present problem. The oxygen diffusion coefficient in the $\mathrm{Ag}$ matrix $D_{\mathrm{O}}{ }^{1}$ takes a value of $2.1 \times 10^{-11} \mathrm{~m}^{2} \mathrm{sec}^{-1}$ at $773 \mathrm{~K}$ [3]. If we assume for convenience that this is the highest diffusion coefficient in the problem, then by putting the corresponding inter-lattice site transition rate $w_{1}$ equal to unity then, from Eq. 10, this immediately means that the basic mesh size $a$ of the lattice is then $2.5 \times 10^{-7} \mathrm{~m}$. In principle, all distances in the problem must then be related to this one. For example, if we wish the shortest distance from the source to the leading edge of the first inclusion to be, say, $0.005 \mathrm{~mm}$ then one would need about 20 lattice spacings of the lattice between the source and the leading edge of the inclusion to achieve this.

Now let us discuss the meaning of time in the present problem. From the above value for the diffusion coefficient of oxygen in the $\mathrm{Ag}$ matrix and the lattice jump distance $a$ we can easily see from Eq. (10) that the basic time unit in the problem is $0.744 \times 10^{-3} \mathrm{sec}$. If we recognize that an attempt to jump per particle is such a time unit; then, for example, 1000 of such time units corresponds to 0.744 seconds of real time.

Finally, let us consider the problem of the width of the interface $\Omega_{2}$. In preliminary work, the interface region $\Omega_{2}$ was treated as rows of lattice sites within the basic lattice. This presents a problem by, in effect, requiring that the interface to be at least the same width as the mesh size $a$. In general, this would make interface unphysically wide. To avoid this, $\Omega_{2}$ was later treated as a set of special virtual sites that do not appear formally in the lattice itself but can be accessed only via the lattice sites immediately around the inclusion. In this way, the width of the interface does not need to be specified or implied.

\section{Results and Discussion}

First, we mention that in the absence of inclusions the simulated concentration profiles from both finite element and Monte Carlo methods were in excellent agreement with the well-known complementary error function solution for diffusion into an infinite medium from a constant source of diffusant at the surface [6].

In Fig. 2a we show an example of an averaged concentration profile for the case where there is no segregation and the inclusions simply are impenetrable to oxygen. Fig. $2 b$ gives the corresponding 2D concentration profile. The behaviour is as expected with a drop in the profile where the impenetrable inclusion impedes the flux of oxygen atoms. In Fig. 3a an example of an averaged concentration profile is given where a segregation factor $s$ of $10^{3}$ has been assumed and in Fig. $3 \mathrm{~b}$ the corresponding $2 \mathrm{D}$ concentration profile is given. The maxima in concentration in both figures correspond to oxygen that has segregated to the interface region. It is noted in Fig. $3 \mathrm{~b}$ that the corners of the interface region have a much higher concentration than elsewhere. This would appear to result from the fact that in the lattice model the corner sites of the interface region have 
two neighbouring sites that can provide oxygen to them whereas other interface sites have only one such provider site. It is quite possible that the effect seen is an artefact of the lattice model being used here to model segregation and that a superior way to model segregation is to invoke the local virtual interface sites as suggested at the end of the previous section where this problem would not occur. In Fig. 3c we present 2D concentration profile with calculations being done with the use of virtual interface sites. In the implementation of this method we effectively added one more coordination direction to all sites to keep the time steps equally spaced. Therefore Eq. 10 should be used with a factor of 5 not 4 .

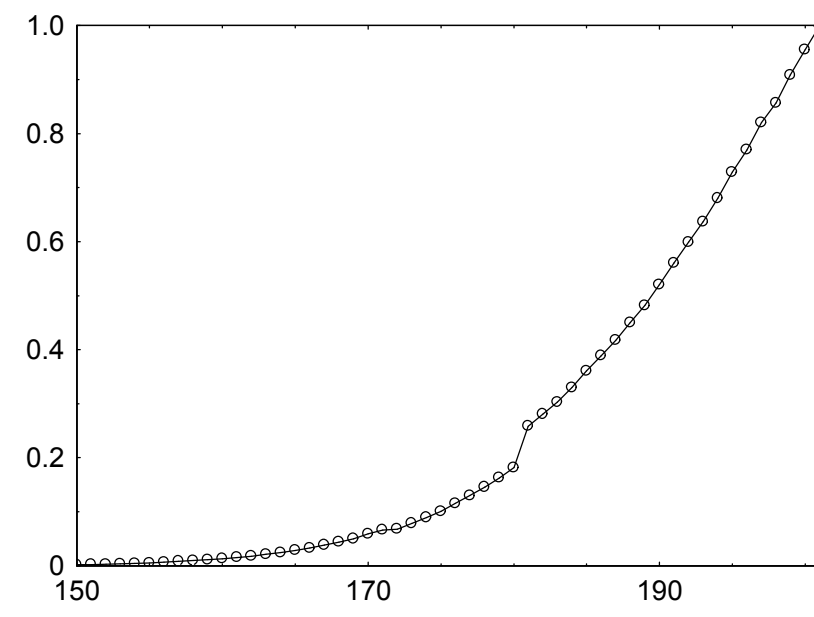

$\psi_{0} / \psi_{0}(201)$

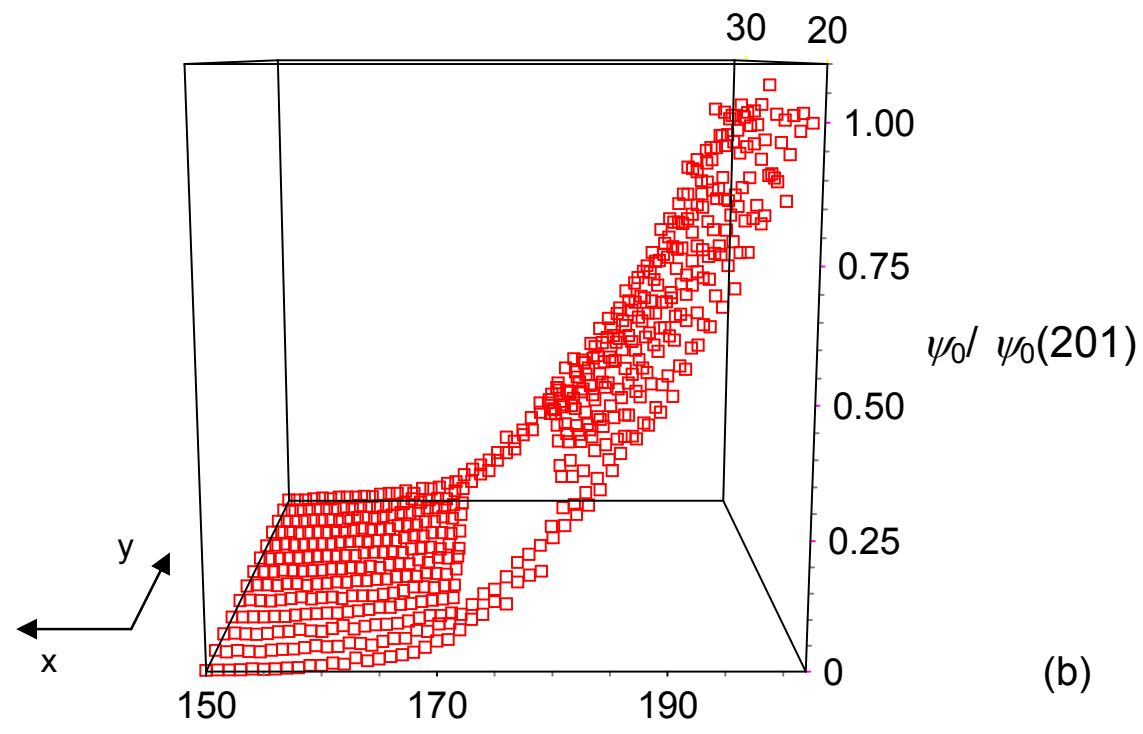

Figure 2. (a) A typical oxygen concentration profile calculated by Monte Carlo method and averaged in the y-direction $(\mathrm{y}=1-50, \mathrm{x}$-direction is shown as lattice plane number). No segregation effect $(s=1.0)$. Total number of jump attempts: $10^{9}$, real time: $0.4166 \mathrm{sec}$., diffusivity in the matrix: $2.1 \times 10^{-11} \mathrm{~m}^{2} \mathrm{sec}^{-1}$, inclusion fraction: 0.0324 . (b) The $2 \mathrm{D}$ concentration profile for the same conditions $(y=19-34)$. 
To test our Monte Carlo simulations we made a series of finite element calculations in parallel with the Monte Carlo simulations for the model described above. Results of this comparison are presented in Figures 4-5. In Fig. 4 we plot $\psi_{O}^{1}$ as a function of $\mathrm{x}$ at $\mathrm{y}=25$ for two times $1.2929 \mathrm{~s}$ and $5.0456 \mathrm{~s}$. We can see that agreement between these two types of calculation for the matrix phase is quite good. For the segregation layer the situation is a bit more complicated. In Table 1 below we present a few point values for $\psi_{O}^{2}$ in the segregation layer calculated by finite element and Monte Carlo analysis. It can be seen that the discrepancies here are between 2 and 14\%. This could be due to the fact that the segregation layer is essentially a singular region in the model. While this can be handled quite easily in the Monte Carlo simulations, in the finite element implementation this introduces significant difficulties.

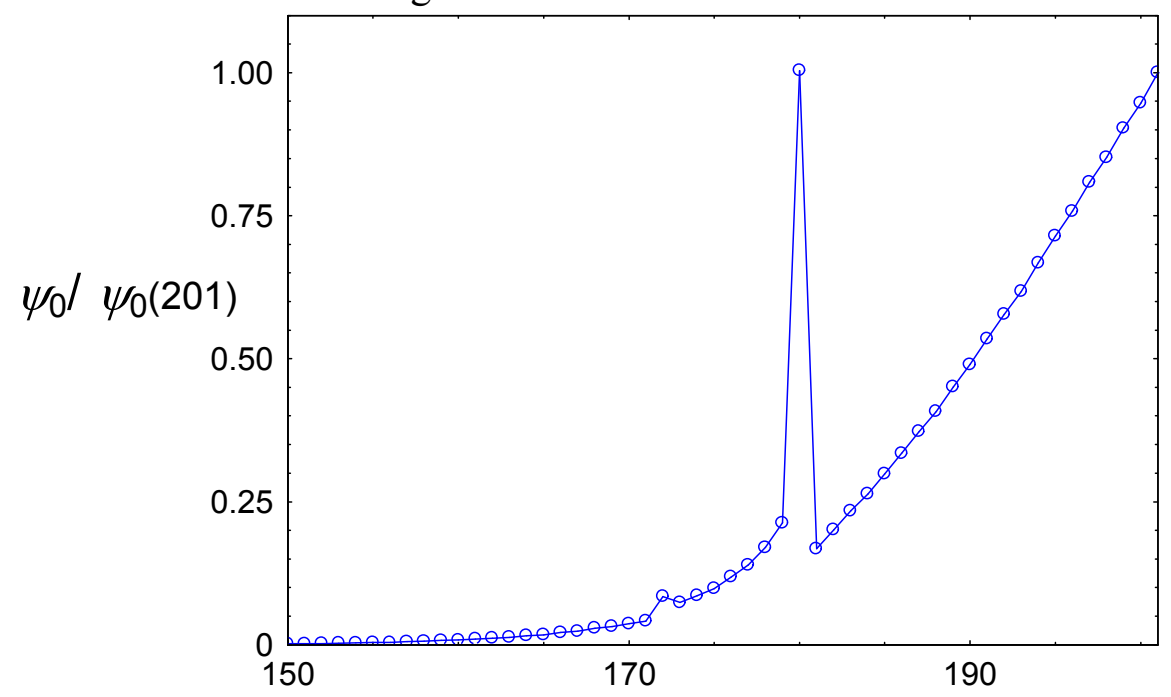

(a)

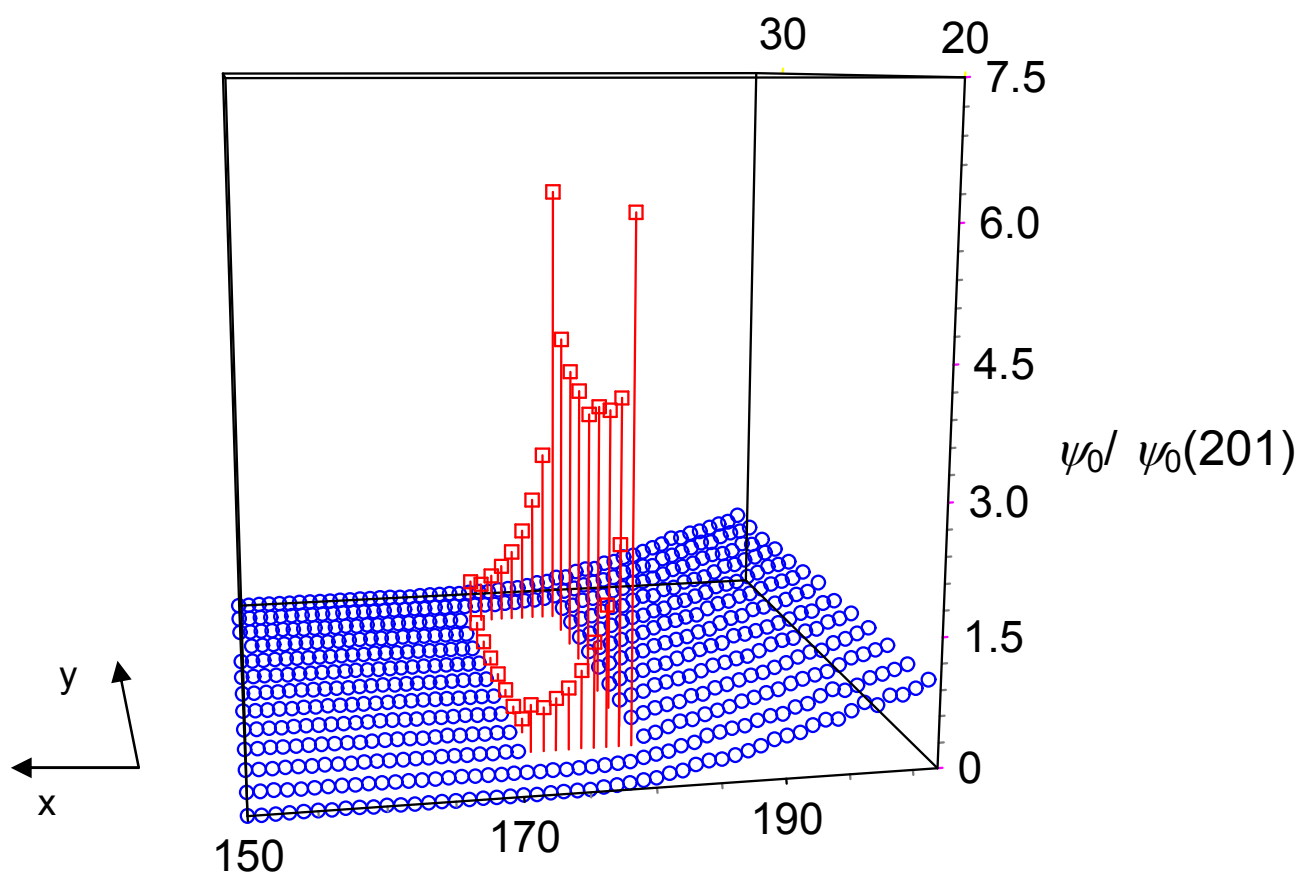

(b) 


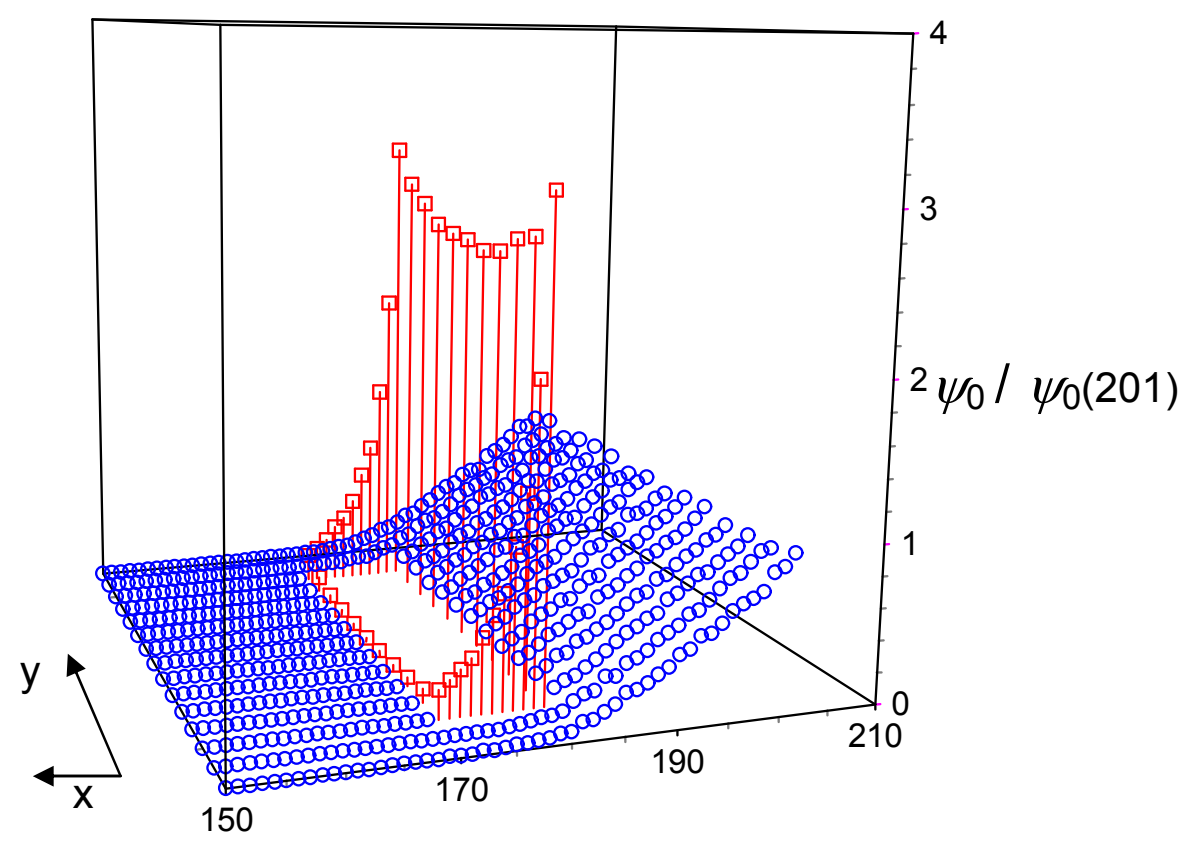

(c)

Figure 3. (a) A typical concentration profile calculated by Monte Carlo method and averaged in ydirection ( $y=1-50, x$-direction is shown as lattice plane number). Segregation factor $\mathrm{s}=10^{3}$, total number of jump attempts: $10^{9}$, real time: $0.4166 \mathrm{sec}$., oxygen diffusivity in the matrix: $2.1 \times 10^{-11}$ $\mathrm{m}^{2} \mathrm{sec}^{-1}$, inclusion fraction: 0.0324 . (b) The $2 \mathrm{D}$ concentration profile for the same conditions $(\mathrm{y}=$ 19 - 34). (c) The 2D concentration profile calculated by Monte Carlo method with the virtual sublattice used, real time: $0.3594 \mathrm{sec}$, all other parameters are the same as in the case (b).

Table of oxygen compositions at the segregation layer

\begin{tabular}{|l|l|l|l|}
\hline Time $(\mathrm{s})$ & $\mathrm{x}($ comp. $)$ & $\psi_{0}^{2}(\mathrm{x})(\mathrm{FE}$ analysis $)$ & $\psi_{0}^{2}(\mathrm{x})(\mathrm{MC}$ simulation $)$ \\
\hline 1.2929 & 21 & 3.4263 & 2.9541 \\
\hline 1.2929 & 29 & 28.6647 & 27.8290 \\
\hline 5.0456 & 21 & 28.1135 & 25.8008 \\
\hline 5.0456 & 29 & 134.9105 & 120.5191 \\
\hline
\end{tabular}

Table 1. Comparison of the point values of $\psi_{O}^{2}$ at $\mathrm{y}=25$ obtained by finite element and Monte Carlo methods.

In Fig. 5 we plot composition profile in 2D segregation layer calculated by means of finite element method. The set of parameters here is analogous to that used in Monte Carlo calculation presented in Fig. 3c. It can be seen that quantitative agreement for the oxygen composition in the segregation layer calculated by means of finite element and Monte Carlo methods is good. 


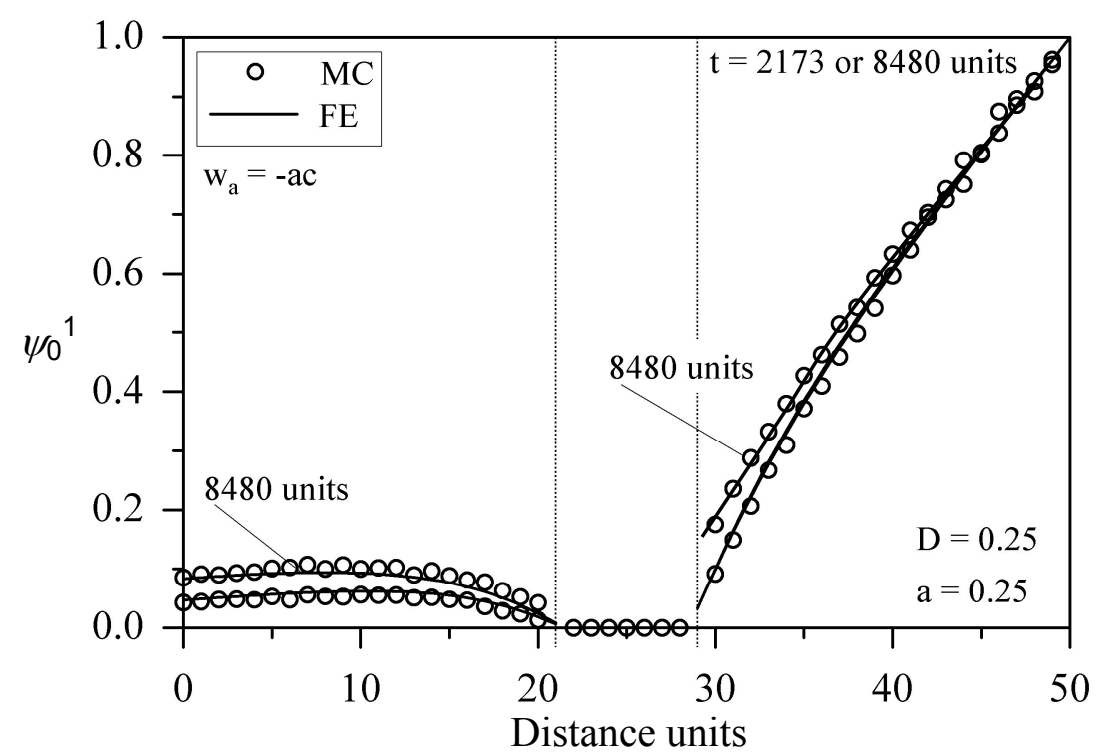

Figure 4. Distribution of $\psi_{O}^{1}$ in the matrix material across the sample at $\mathrm{y}=25$. Solid lines represent the finite element analysis calculation; symbols represent the Monte Carlo simulation results. Time in time units corresponds to time in seconds as follows: 2173 time units is equal to 1.2929 seconds; 8480 time units is equal to 5.0456 seconds.

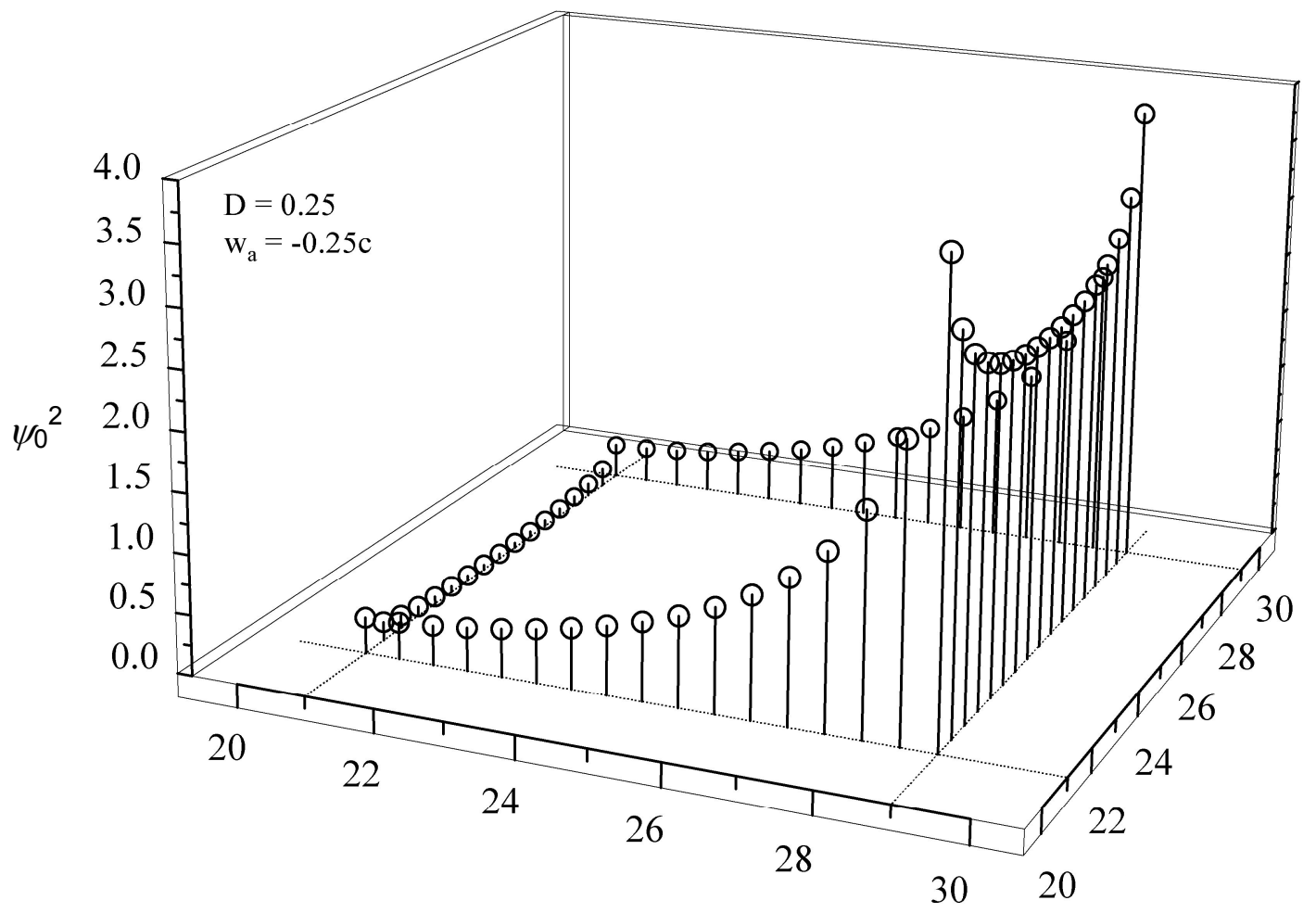

Figure 5. The 2D segregation layer profile calculated by the finite element method, real time: $0.3594 \mathrm{sec}$, segregation factor $\mathrm{s}=10^{3}$, oxygen diffusivity in the matrix: $2.1 \times 10^{-11} \mathrm{~m}^{2} \mathrm{sec}^{-1}$. This profile to compare with segregation layer region (squares) presented in Fig. 3c. 


\section{Summary}

The problem of oxygen segregation at the internal metal-ceramic interfaces have been addressed for the model system $\mathrm{Ag}-\mathrm{MgO}$ from a phenomenological aspect using a lattice-based Monte Carlo method and a finite element method extended with special user-subroutines. We have simulated the time-dependence of oxygen depth and contour profiles and the segregation layer of the coveragetype composition function for oxygen. We have been able to show very good agreement between these two methods for the matrix phase. For the oxygen coverage of the segregation layer a quantitative agreement between these two methods have been observed. Discrepancy in point values for the $\mathrm{FE}$ and $\mathrm{MC}$ calculations have been estimated as under $14 \%$ and on average as $8 \%$.

The lattice-based Monte Carlo method has been demonstrated here to have the capability to address the problem of diffusion of oxygen into a $A g-M g O$ composite with accompanying segregation of oxygen at the $A g-M g O$ interfaces. In the future, it is anticipated that the lattice Monte Carlo method will be used to address this problem using more realistic sizes/shapes and distributions (e.g. random) of the $M g O$ inclusions. (Such refinements present a major difficulty for the finite element method but do not present a difficulty for the lattice Monte Carlo method.) It is also anticipated that the lattice-based Monte Carlo method will be used to address the problem of out-diffusion where, at higher temperatures, oxygen leaves the interfaces and matrix of the composite in a diffusion-limited evaporation process.

\section{Acknowledgements}

We wish to thank the Australian Research Council (IVB and GEM) and the Portuguese Foundation of Science and Technology (AÖ) for their support of this research.

\section{References}

[1] M. Stasiek, A. Öchsner and J. Grácio: Defect Diffusion Forum. Vol. 249 (2006), p.35.

[2] I. Kaur, Y. Mishin and W. Gust, in Fundamentals of Grain and Interphase Boundary Diffusion, (Wiley; Chichester) 1995.

[3] M. Stasiek, A. Öchsner and J. Grácio: J. Phase Equilibria and Diffusion, Vol. 27 (2006), p.644.

[4] G.E. Murch and I.V. Belova: Interface Science, Vol. 11 (2003), p.91.

[5] I.V. Belova and G.E. Murch: in Cellular and Porous Materials. Thermal Properties Simulation and Prediction, edited by A. Öchsner, G.E. Murch and J.S. de Lemos (Wiley - VCH; Weinheim) in press.

[6] J. Crank, The Mathematics of Diffusion. (Clarendon Press; Oxford), 1975. 
Diffusion in Advanced Materials and Processing

doi:10.4028/3-908451-45-0

Modelling of Oxygen Diffusion and Segregation at Interfaces in Ag-MgO Composites

doi:10.4028/3-908451-45-0.29 